\title{
Weakly bound diquarks and Efimov hyperons in QCD*
}

T. Frederico, J. A. O. Marinho, E. Gambin

Instituto Tecnológico de Aeronáutica, São José dos Campos, Brasil

PLAN:

Motivation: Diquarks, Hadron Spectrum, Nucleon SL EM form factors QCD Model with color antriplet-scalar diquark

Running coupling constant - Fixed point

Gluon and photon in-medium masses

Zero-binding diquarks \& Thomas-Efimov physics

Efimov hyperons and delta-like Conclusion

${ }^{*}$ Continuous Advances in QCD, U. Minnesota, 2006 


\section{Motivation Diquarks}

R. L. Jaffe, Nucl. Phys. Proc. Suppl 142 (2005) 343; Phys.Rep. 409 (2005) 1

$$
|p\rangle=|q q q\rangle+|q q q q \bar{q}\rangle+|q q q g\rangle \ldots
$$

Quark-quark color/flavor IRep. $\quad 3 \otimes 3=6 \oplus \overline{3}$

$$
\begin{aligned}
& \left|(q q)(\overline{3})_{c}(A)(\overline{3})_{f}(A)\left(0^{+}\right)(A)\right\rangle \\
& \left|(q q)(\overline{3})_{c}(A)(6)_{f}(S)\left(1^{+}\right)(S)\right\rangle
\end{aligned}
$$
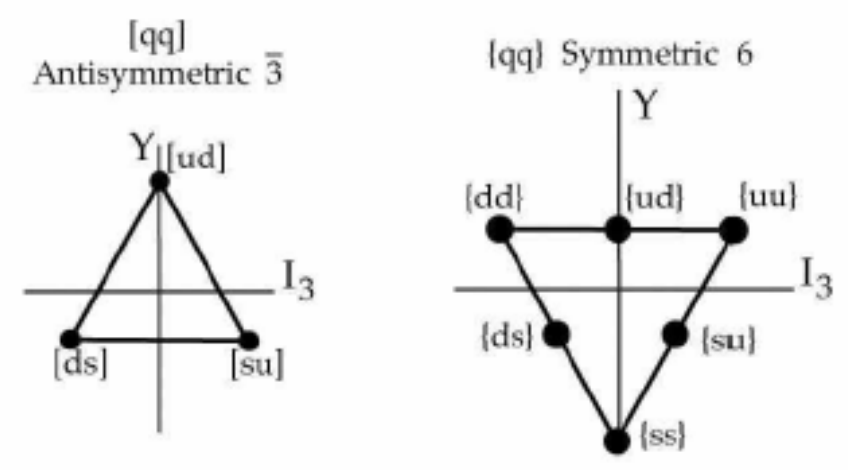

$\mathbf{N}(938) \quad \Delta(1232) I\left(J^{P}\right)=3 / 2(3 / 2)^{+}$ 
Scalar diquarks: diquark-antidiquark system

Singlet \& octect exotic mesons

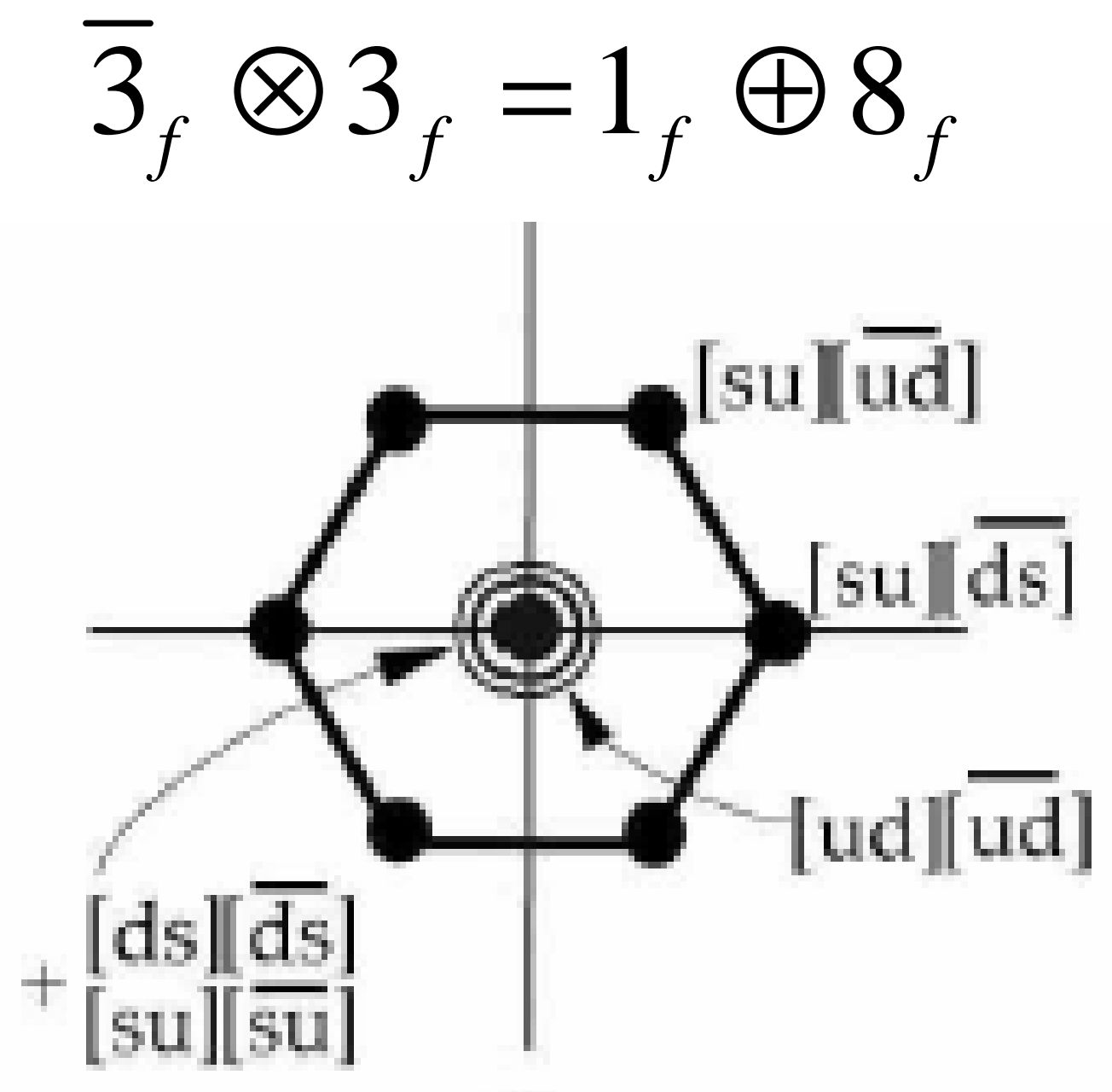




\section{Tetraquark nonet}

L. Maiani et.al., "New look to scalar mesons", Phys. Rev. Lett. 93 (2004) 212002;

TABLE I. Experimental values for the salar meson masses

\begin{tabular}{ccc}
\hline \hline Meson & Mass (MeV) & Source \\
\hline$\sigma$ & $478 \pm 24 \pm 17$ & {$[7]$} \\
$\kappa$ & $797 \pm 19 \pm 43$ & E791 [8] \\
$f$ & $980 \pm 10$ & PDG [2] \\
$a$ & $984.7 \pm 1.2$ & PDG [2] \\
\hline \hline
\end{tabular}

[7] KLOE Collaboration, A. Aloisio et al, Phys Lett. B 537, 21 (2002); E. M. Aitala et al, Phys Rev. Lett. 86, 770 (2001)

[8] E. M. Aitala et al, Phys. Rev. Lett. 89, 121801 (2002) 


\section{Hadron spectrum}

Chew-Frautschi formula

$$
M^{2}=a+\sigma L
$$

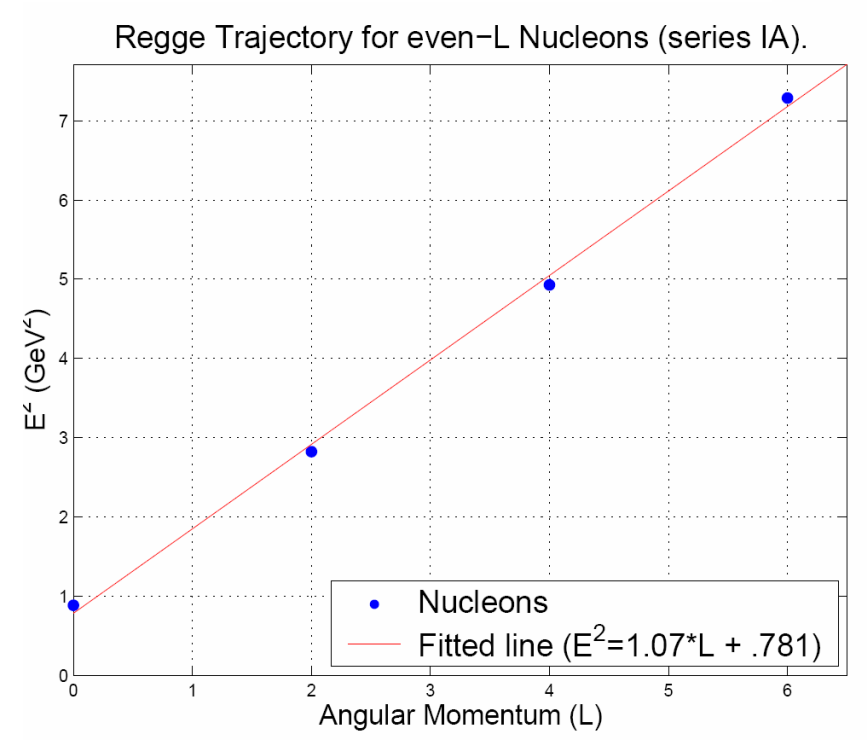

Diquarks as Inspiration and as Objects

Frank Wilczek*

hep-ph/0409168

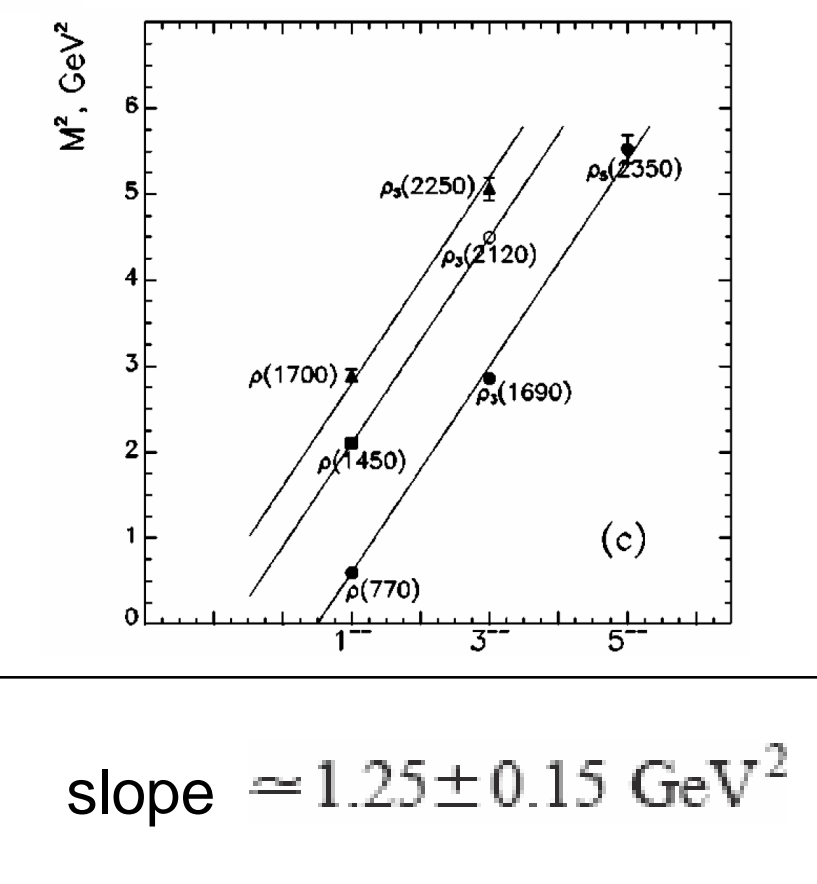

PHYSICAL REVIEW D, VOLUME 62, 051502(R)

Systematics of $q \bar{q}$ states in the $\left(n, M^{2}\right)$ and $\left(J, M^{2}\right)$ planes

A. V. Anisovich, V. V. Anisovich, and A. V. Sarantsev 


\section{Nucleon SL EM form factors}

$$
\begin{aligned}
\mathcal{L}_{\mathrm{N}-3 \mathrm{q}}= & \alpha m_{\mathrm{N}} \epsilon^{l m n} \bar{\Psi}_{(l)} \mathrm{i} \tau_{2} \gamma^{5} \Psi_{(m)}^{\mathrm{C}} \bar{\Psi}_{(n)} \Psi_{\mathrm{N}} \\
& +(1-\alpha) \epsilon^{l m n} \bar{\Psi}_{(l)} \mathrm{i} \tau_{2} \gamma_{\mu} \gamma^{5} \Psi_{(m)}^{\mathrm{C}} \bar{\Psi}_{(n)} \mathrm{i} \partial^{\mu} \Psi_{\mathrm{N}}+\text { H.C. }
\end{aligned}
$$

Light-front model of the nucleon wave function

E.F. Suisso et al. / Nuclear Physics A 694 (2001) 351-371

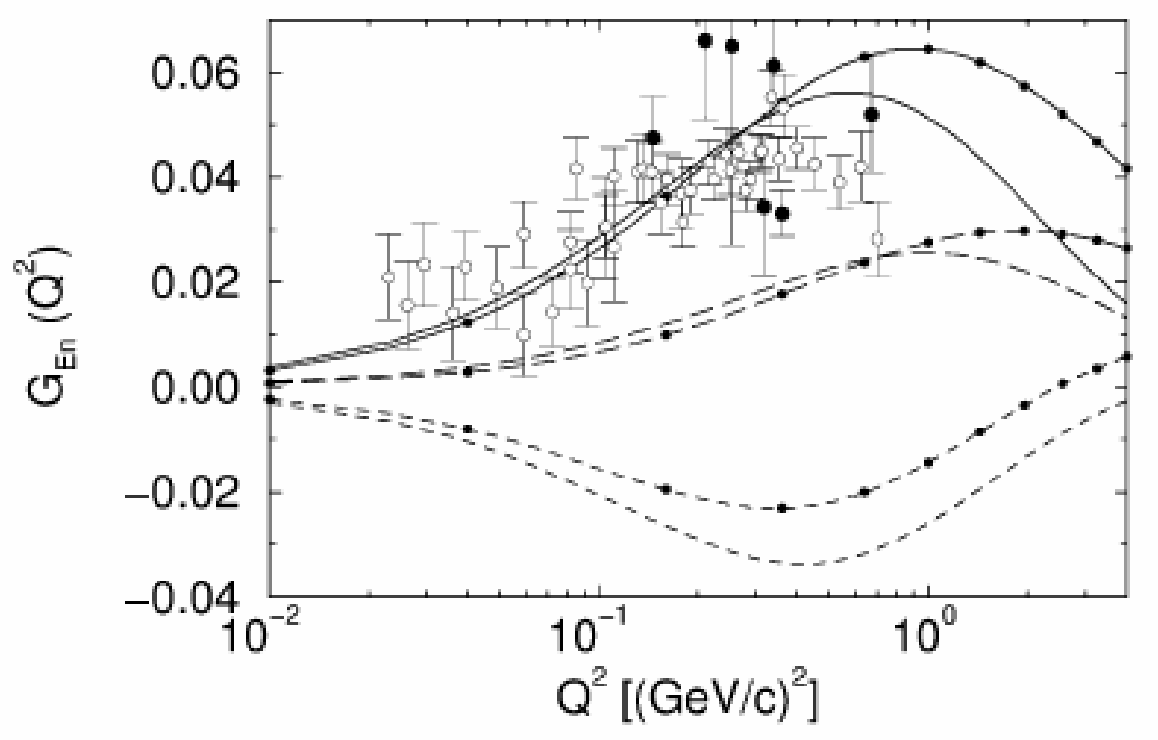

solid $\quad \alpha=1$

dashed $\alpha=1 / 2$

dotted $\quad \alpha=0$ 


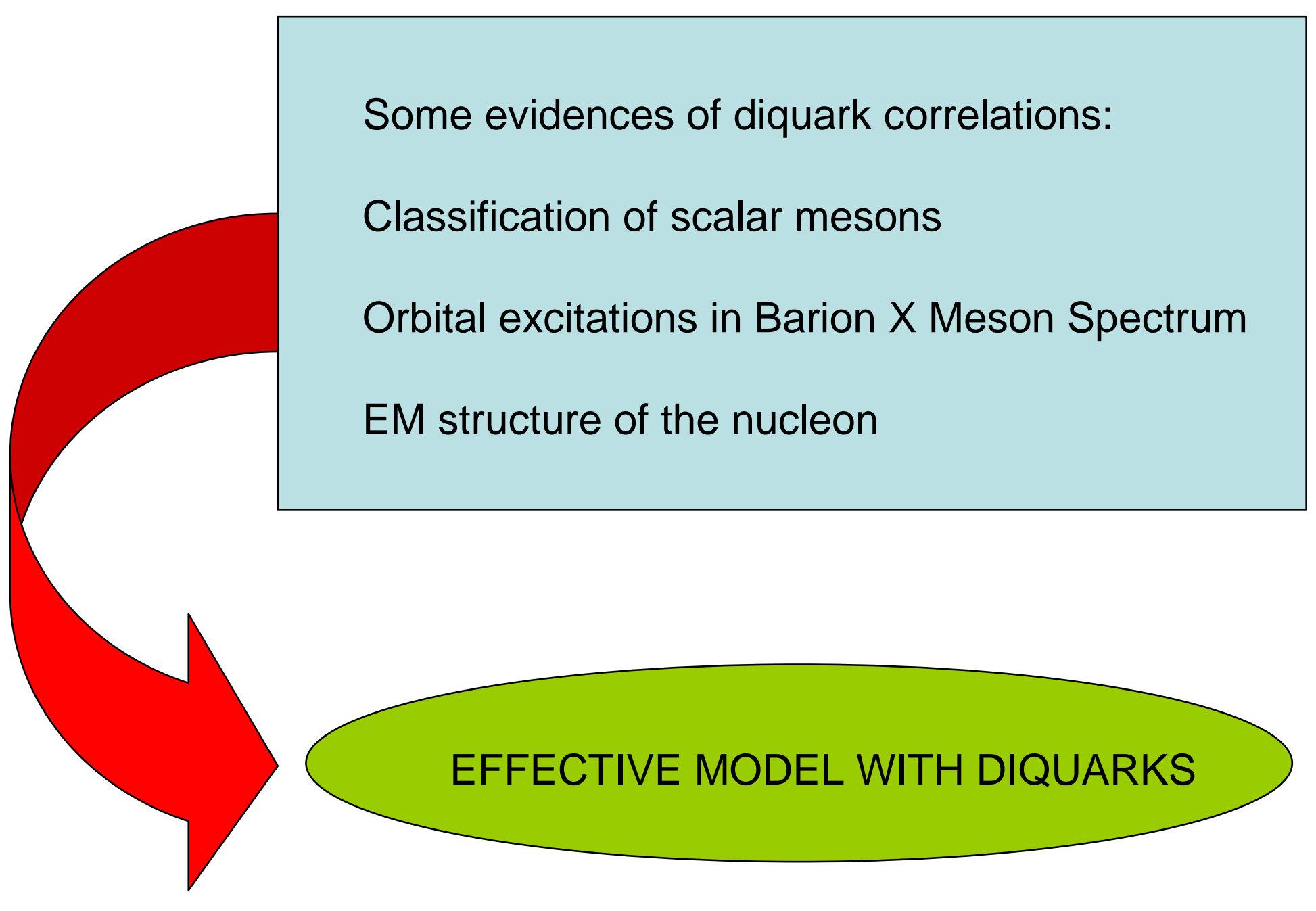




\section{QCD Model with color antriplet-scalar diquark}

Hong, Sohn, Zahed Phys. Lett. B596 (2004) 191

$L=-\frac{1}{4} G^{a, \mu \nu} G_{\mu \nu}^{a} \overline{+} \bar{\Psi}\left(\imath D_{(q)}-m_{q}\right) \Psi+\left(D_{(s)}^{\mu} \phi\right)^{\dagger}\left(D_{(s) \mu} \phi\right)-\phi^{\dagger} \hat{m}_{s}^{2} \phi$

$\left(D_{(s) \mu} \phi\right)_{i}=\partial_{\mu} \phi_{i}-i g A_{\mu}^{a}\left(T_{i j}^{a}\right)^{*} \phi_{j} \quad\left(D_{(q) \mu} \psi\right)_{i}=\partial_{\mu} \psi_{i}+i g A_{\mu}^{a} T_{i j}^{a} \psi_{j}$

$G_{\mu \nu}^{a}=\partial_{\mu} A_{\nu}^{a}-\partial_{\nu} A_{\mu}^{a}-g f^{a b c} A_{\mu}^{b} A_{\nu}^{c}$ 

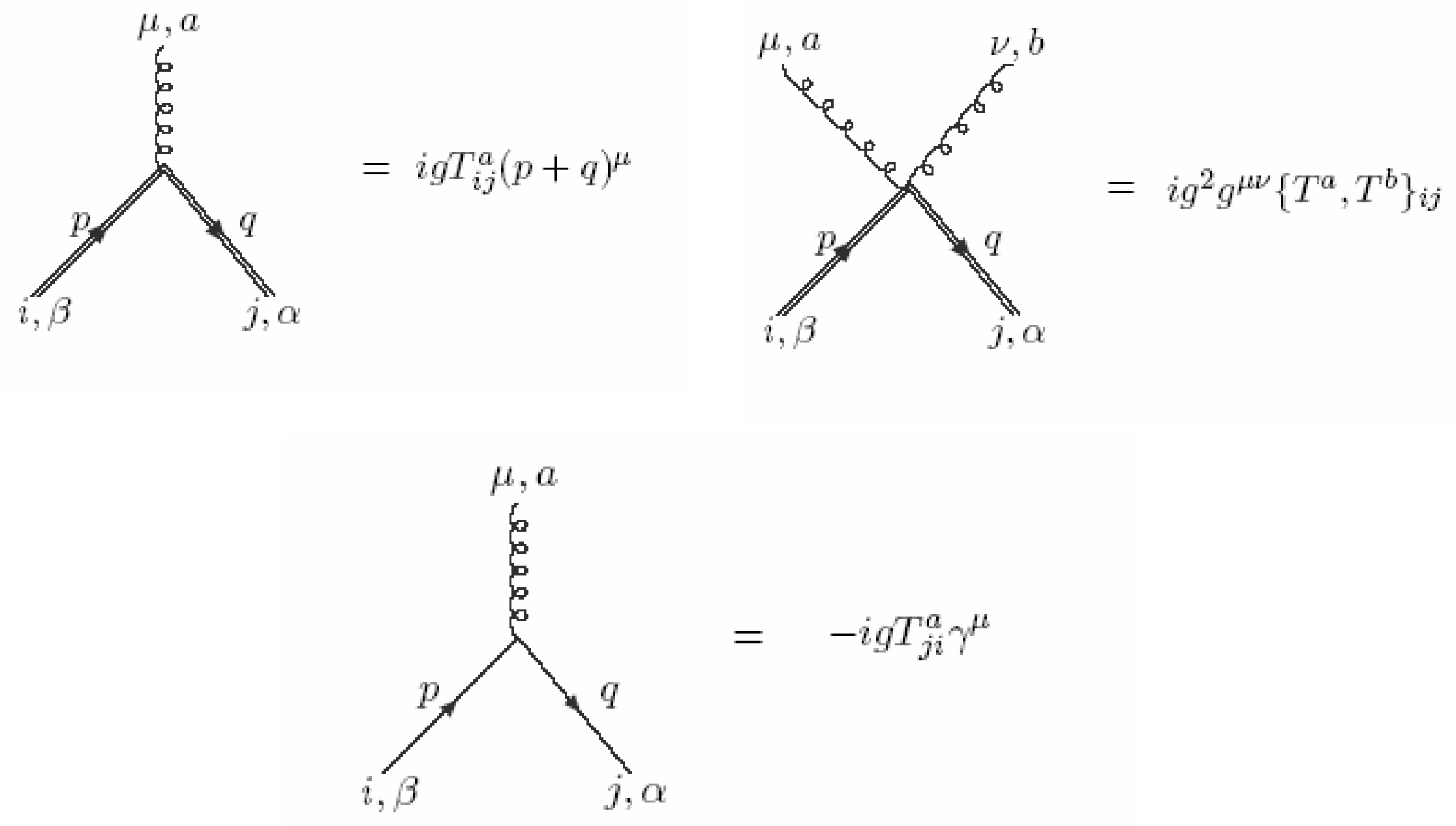

$$
=-i g T_{j i}^{a} \gamma^{\mu}
$$




\section{Running coupling constant}

$$
\Psi=\sqrt{Z_{2}} \Psi_{B}, A_{B \mu}^{a}=\sqrt{Z_{3}} A_{\mu}^{a}, \phi_{B}=\sqrt{Z_{2}^{s}} \phi_{B}
$$

$$
g_{B}=g \mu^{\frac{\epsilon}{2}} \frac{Z_{1}}{Z_{2} \sqrt{Z_{3}}}=g \mu^{\frac{\epsilon}{2}} \frac{Z_{1}^{s}}{Z_{2}^{s} \sqrt{Z_{3}}}=\ldots
$$

$$
\frac{d g_{B}}{d \mu}=0
$$




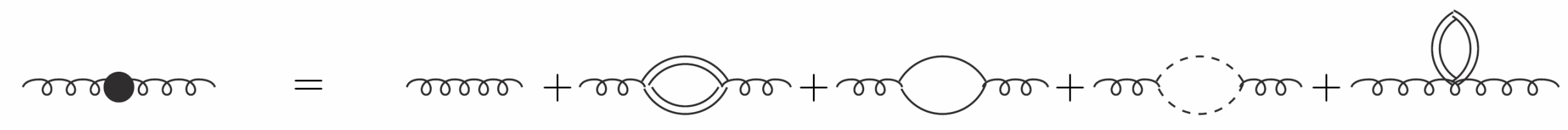

$$
\Pi_{a b}^{\mu \nu}(d 1+d 2)=\frac{g^{2}}{48 \pi^{2} \varepsilon} N_{s} \delta_{a b}\left[\left(p^{\mu} p^{\nu}-p^{2} g^{\mu \nu}\right)\right]
$$

Ns number of diquark flavors 


$$
Z_{3}=1+\frac{g^{2}}{8 \pi^{2} \varepsilon}\left(5-\frac{2}{3} N_{q}-\frac{1}{6} N_{s}\right)
$$

$$
\alpha_{Q C D}^{q+s}\left(Q^{2}\right)=\frac{4 \pi}{\left(11-\frac{2}{3} N_{q}-\frac{1}{6} N_{s}\right) \log \frac{Q^{2}}{\Lambda^{2}}}
$$




\section{QCD FIXED-POINT}

$$
\beta(g)=-\frac{g^{3}}{(4 \pi)^{2}}\left(11-\frac{2}{3} N_{q}-\frac{1}{6} N_{s}\right)
$$

only $[q q]^{\overline{3}_{c}}$ configurations $\quad N_{s}=N_{q}\left(N_{q}-1\right) / 2$

$$
11-\frac{2}{3} N_{q}^{*}-\frac{1}{12} N_{q}^{*}\left(N_{q}^{*}-1\right)=0
$$

$N_{q}^{*} \approx 8.5$

freezing of the coupling constant near the physical flavor number!

T. Banks and A. Zaks, Nucl. Phys. B196 (1982) 189

P. M. Stevenson, Phys. Lett. B331 (1994) 187 


\section{Gluon and photon in-medium masses}

$$
L_{A^{2}}=g^{2} A^{\mu a} A_{\mu}^{b} \phi_{j}^{\dagger}\left(T^{a}\right)_{i j} T_{i k}^{b_{i k}^{*}} \phi_{k}
$$

$$
m_{g}^{2}=2 g^{2}\left\langle\phi_{j}^{\dagger}\left(T^{a}\right)_{i j} T_{i k}^{a *} \phi_{k}\right\rangle
$$

colorless medium

$$
\left\langle\phi^{\dagger} \phi\right\rangle=\frac{1}{3} \sum_{\text {flavor }, i}\left\langle\phi_{i}^{f \dagger} \phi_{i}^{f}\right\rangle
$$

$$
m_{g}^{2}=2 g^{2}\left\langle\phi^{\dagger} \phi\right\rangle\left(T^{a}\right)_{i j} T_{i j}^{a *}=4 g^{2}\left\langle\phi^{\dagger} \phi\right\rangle
$$


Estimative of in-medium gluon mass

$$
\begin{gathered}
m_{g}^{2}=2 g^{2}\left\langle\phi^{\dagger} \phi\right\rangle\left(T^{a}\right)_{i j} T_{i j}^{a *}=4 g^{2}\left\langle\phi^{\dagger} \phi\right\rangle \\
\sum_{\text {flavor }, i} m_{s, f}^{2}\left\langle\phi_{i}^{f \dagger} \phi_{i}^{f}\right\rangle \sim \varepsilon \\
\varepsilon_{0}=1 \mathrm{GeV} / \mathrm{fm}^{3}
\end{gathered}
$$

- $\alpha_{Q C D} \sim 1 \quad \epsilon=\epsilon_{0} \quad m_{s} \sim 0.6 \mathrm{GeV}$

$$
m_{g} \sim 0.6 \mathrm{GeV}
$$


Estimative of in-medium photon mass

$$
m_{\gamma} \sim \sqrt{e^{2} \varepsilon \sum_{f} q_{f}^{2} /\left(N_{s} m_{s}^{2}\right)} .
$$

few tens of $\mathrm{MeV}$ near the chiral phase transition 


\section{Zero-binding diquarks \& Thomas-Efimov physics}

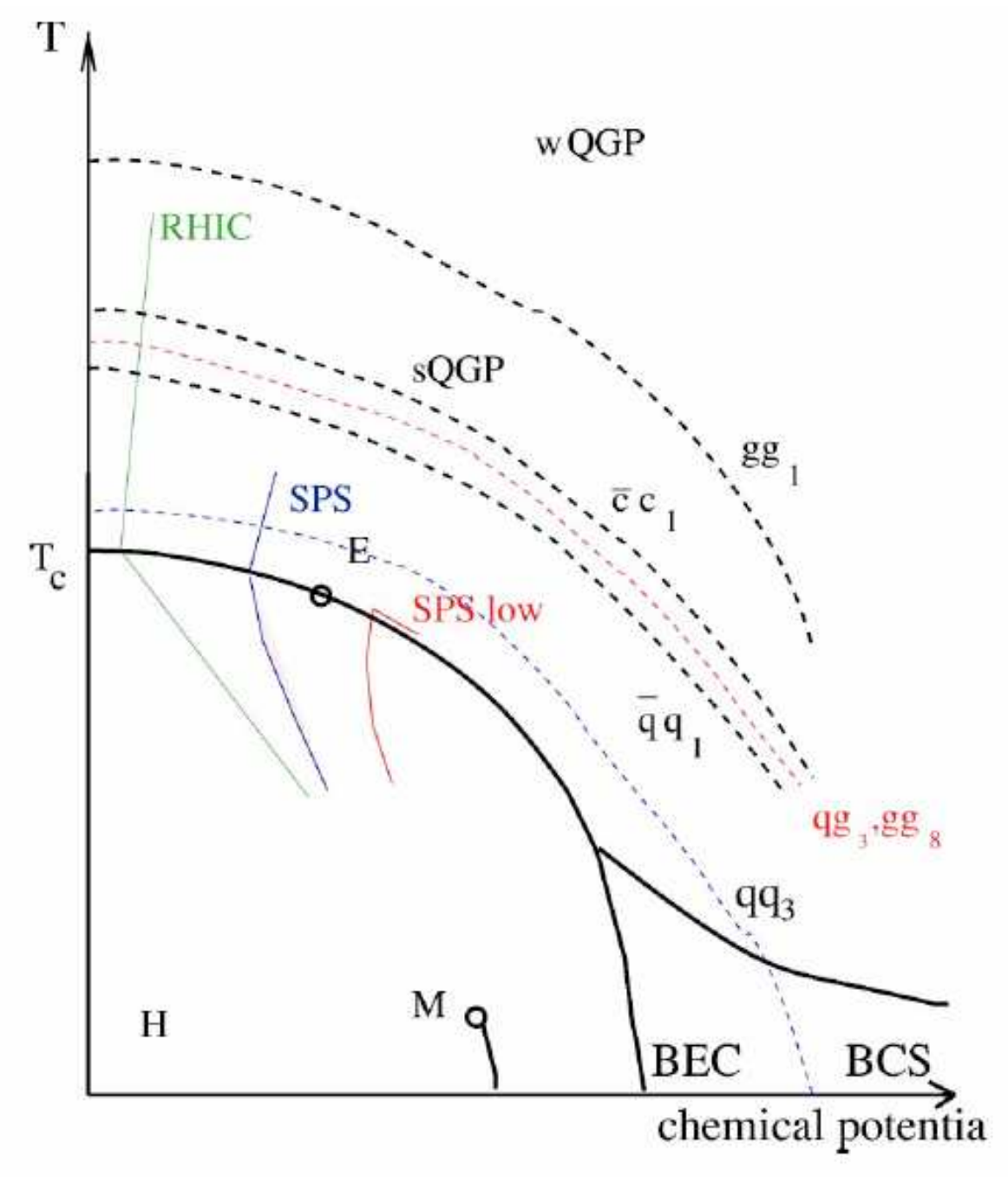

E. V. SHURYAK AND I. ZAHED

PHYSICAL REVIEW C 70, 021901(R) (2004) 
Weakly bound systems \& contact interaction

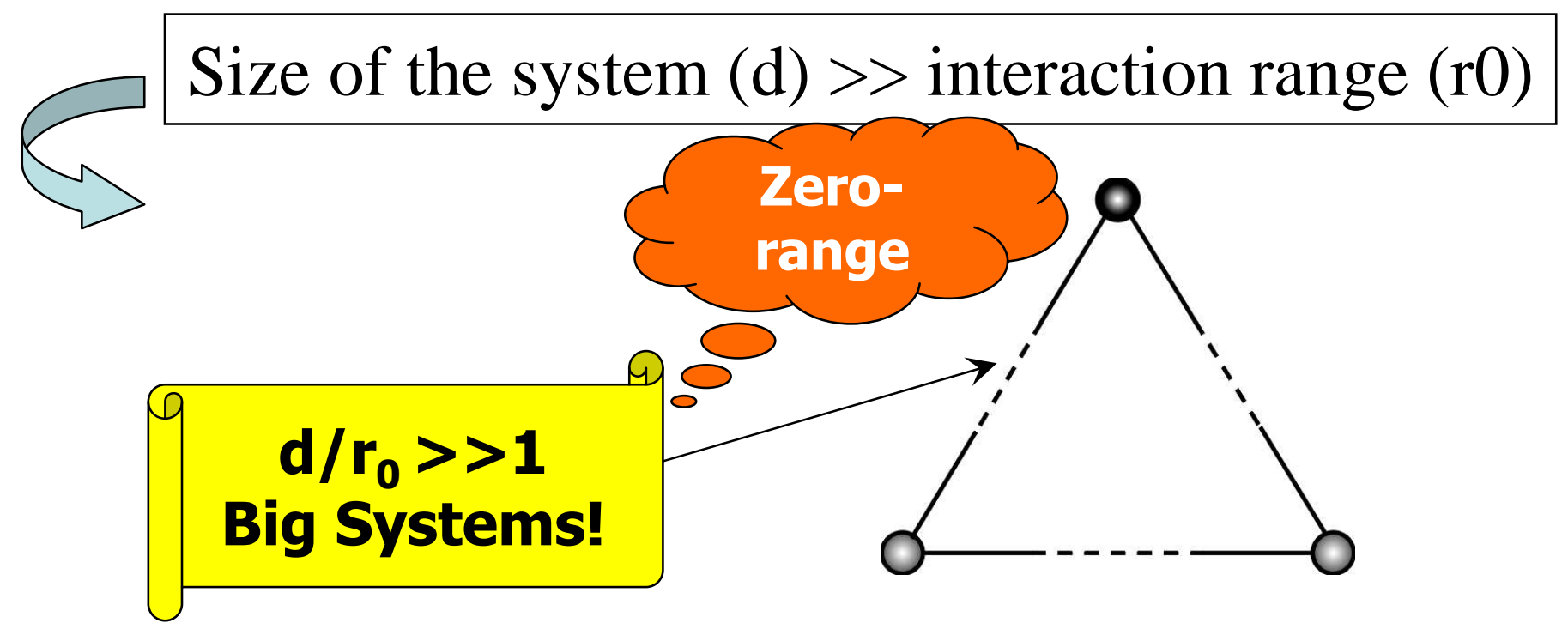

The eigenfunction of the system satisfies a free Schrödinger equation almost everywhere for nonzero interparticle distances!

\section{asymptotic wf behaviour \& universality}

A. S. Jensen, K. Riisager, D. V. Fedorov, and E. Garrido, Rev. Mod. Phys. 76, 215 (2004) 
Weakly bound systems \& contact interaction

Example: Trinucleon quartet state $\mathrm{S}=3 / 2$

$$
\left(E-H_{0}\right) \psi=0
$$

$$
\begin{gathered}
\psi=\int d^{3} q_{1} \frac{\exp \left\{i\left[E-(3 / 4) q_{1}^{2}\right]^{1 / 2} R_{1}\right\}}{R_{1}} e^{i \mathbf{q}_{1} \cdot \mathbf{r}_{1}} \chi\left(\mathbf{q}_{1}\right) \\
-\int d^{3} q_{3} \frac{\exp \left\{i\left[E-(3 / 4) q_{3}^{2}\right]^{1 / 2} R_{3}\right\}}{R_{3}} e^{i \mathbf{q}_{3} \cdot \mathbf{r}_{3}} \chi\left(\mathbf{q}_{3}\right) \\
\text { R_i relative distance between particles j and k } \\
\text { r_i relative distance between i and jk CM }
\end{gathered}
$$


Scale invariance in few-body systems

Speètator function for 3-bosons

$$
a^{-1}= \pm \sqrt{\epsilon_{2}}
$$

$$
\chi(\vec{y})=\frac{-\pi^{-2}}{ \pm \sqrt{\epsilon_{2}}-\sqrt{\epsilon_{3}+\frac{3}{4} \vec{y}^{2}}} \int d^{3} x \frac{1}{\epsilon_{3}+\vec{y}^{2}+\vec{x}^{2}+\vec{y} \cdot \vec{x}} \chi(x)
$$

If $\boldsymbol{\epsilon}_{2}$ and $\boldsymbol{\epsilon}_{3}=0$

Spectator function for a triquark-system

$$
\begin{gathered}
\chi(q)=C_{\kappa} \frac{4}{\sqrt{3} \pi} \int_{0}^{\infty} d k \chi(k) \ln \frac{q^{2}+q k+k^{2}}{q^{2}-q k+k^{2}} \\
\kappa \equiv\left\{S, I \operatorname{Rep}_{c}, I R e p_{f}\right\}
\end{gathered}
$$


Scale invariance: $\chi(\xi y)$ is also solution!!!

$$
\begin{gathered}
\chi(q)=q^{i \pi s_{0}-1} \quad 1=C_{\kappa} \frac{8}{\sqrt{3} s_{0}} \frac{\sinh \frac{\pi s_{0}}{6}}{\cosh \frac{\pi s_{0}}{2}} \\
C_{\kappa}>\frac{3 \sqrt{3}}{4 \pi} \quad\left(s_{0}=0\right)
\end{gathered}
$$

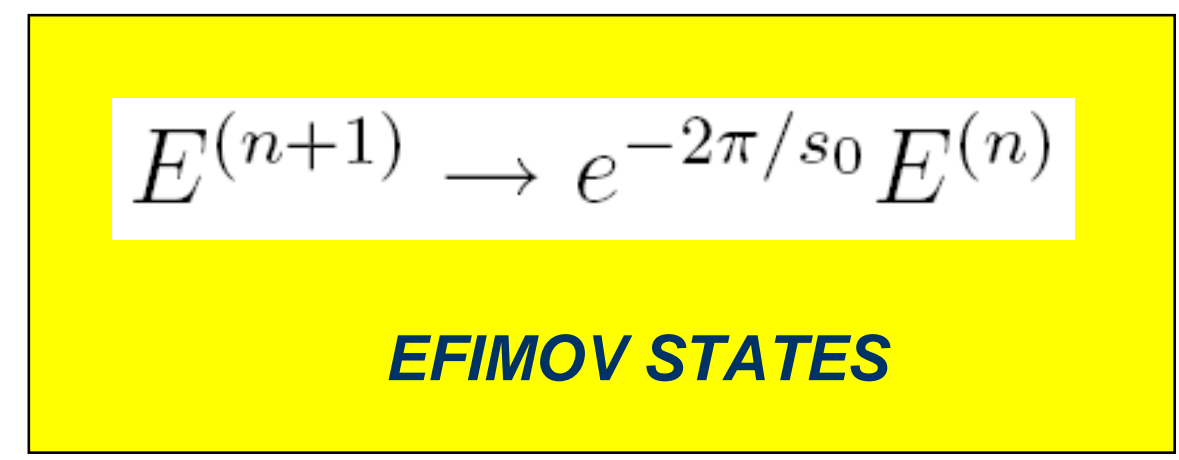

V. Efimov, Phys. Lett. B 33 (1970) 563; Nucl. Phys. A362 (1981) 45; V. Efimov, Comm. Nucl. Part. Phys. 19 (1990) 271.

E. Braaten and H.-W. Hammer, to appear in Physics Reports (cond-mat/0410417) 
Spontaneous breaking of scale invariance\&Thomas-Efimov effect

Nature breaks scale invariance $\rightarrow$ momentum/energy scale

$$
\begin{array}{r}
\chi(\vec{y})=\frac{-\pi^{-2}}{ \pm \sqrt{\epsilon_{2}}-\sqrt{\epsilon_{3}+\frac{3}{4} \vec{y}^{2}}} \int d^{3} x\left(\frac{1}{\epsilon_{3}+\vec{y}^{2}+\vec{x}^{2}+\vec{y} \cdot \vec{x}}-\frac{1}{1+\vec{y}^{2}+\vec{x}^{2}+\vec{y} \cdot \vec{x}}\right) \chi(\vec{x}) \\
\mu_{(3)}^{2}=1 \quad \text { Adhikari,TF,Goldman, PRL74 (1995) } 487
\end{array}
$$

Thomas collapse: $\mu_{(3)}^{2} \rightarrow \infty$

Efimov effect: $\left.\quad E_{2} \rightarrow 0\right\} \epsilon_{2} \rightarrow 0$

S.K. Adhikari, A. Delfino, T. Frederico, I.D. Goldman, and L.

Tomio, Phys. Rev. A 37, 3666 (1988).

$$
\boldsymbol{\epsilon}_{3}^{(N)} \equiv \boldsymbol{\epsilon}_{3}^{(N)}\left( \pm \sqrt{\boldsymbol{\epsilon}_{2}}\right)
$$




\section{Scaling limit \& limit cycle}

$\frac{E_{3}^{(N+1)}}{E_{3}^{(N)}}=\lim _{N \rightarrow \infty} \frac{\epsilon_{3}^{(N+1)}(\xi)}{\epsilon_{3}^{(N)}}=\mathcal{F}\left( \pm \sqrt{\frac{E_{2}}{E_{3}^{(N)}}}\right)$

Scaling limit:

Frederico et al PRA60 (1999)R9

Yamashita et al PRA66(2003)052702

Limit cycle:

R.F. Mohr, R.J. Furnstahl, H.-W. Hammer, R.J. Perry, and K.G. Wilson, Ann. Phys. 321, 225 (2006).
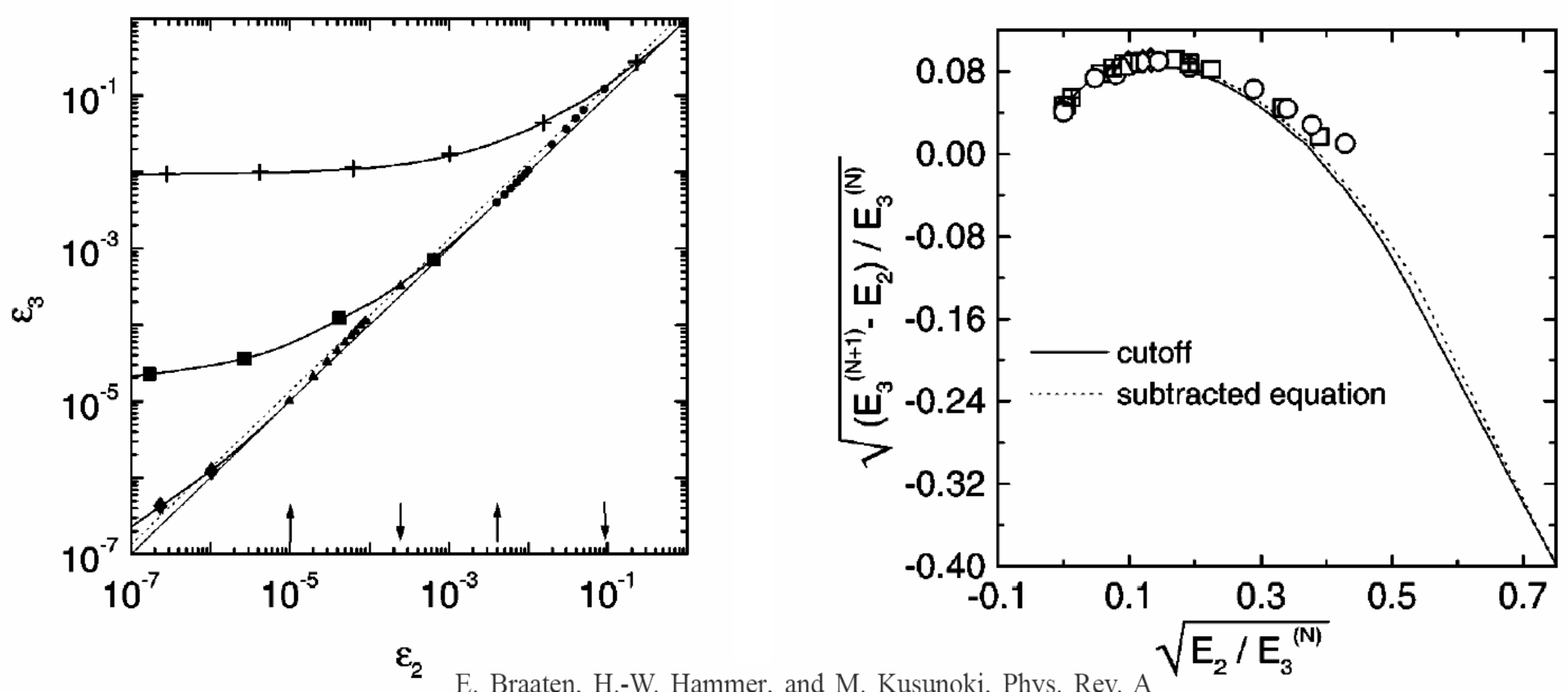

67, 022505 (2003). 


\section{Scaling functions: Correlation between observables}

\section{$S$-wave three-boson resonance}
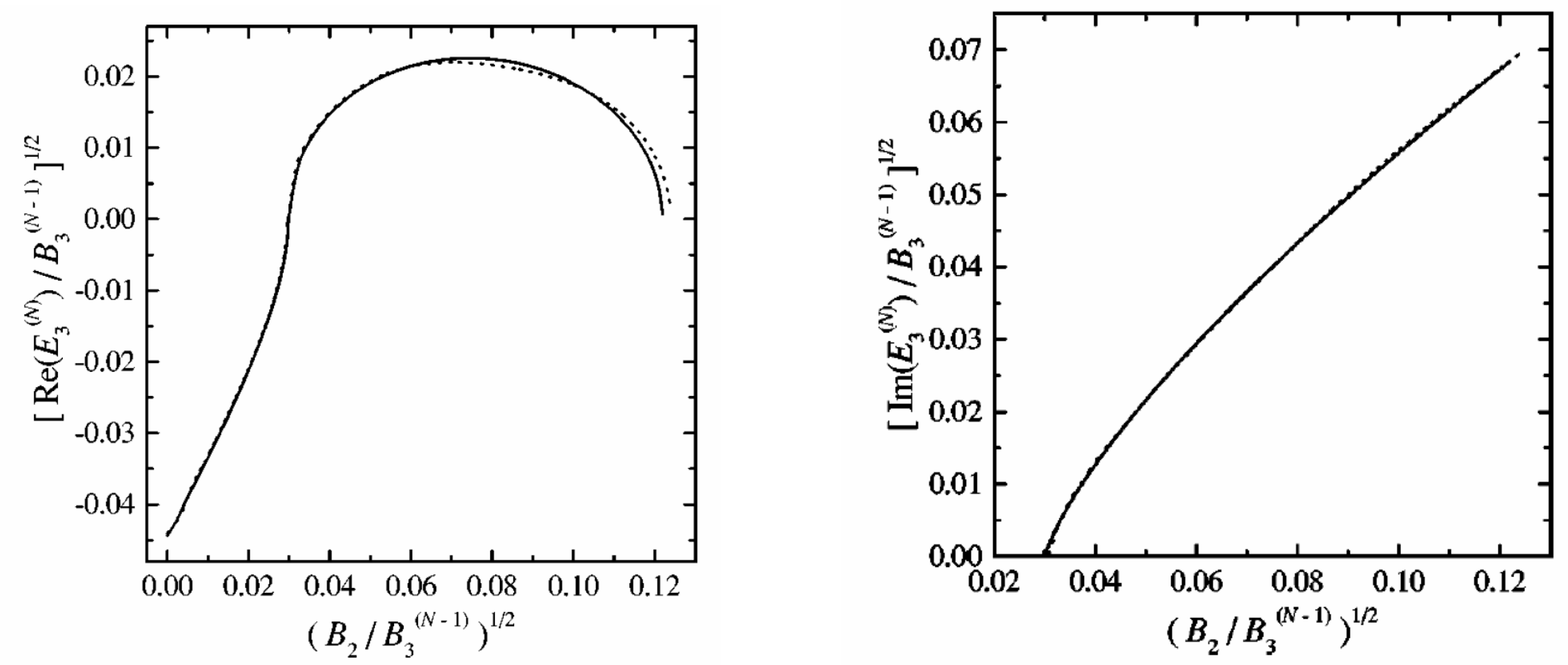

Bringas, Yamashita, TF, PRA69(2004)040702(R) 


\section{Evidence for Efimov quantum states in an ultracold gas of caesium atoms}

T. Kraemer ${ }^{1}$, M. Mark ${ }^{1}$, P. Waldburger ${ }^{1}$, J. G. Danzl ${ }^{1}$, C. Chin ${ }^{1,2}$, B. Engeser ${ }^{1}$, A. D. Lange ${ }^{1}$, K. Pilch ${ }^{1}$, A. Jaakkola ${ }^{1}$, H.-C. Nägerl ${ }^{1} \&$ R. Grimm ${ }^{1,3}$

\section{NATURE|Vol 440|16 March 2006}

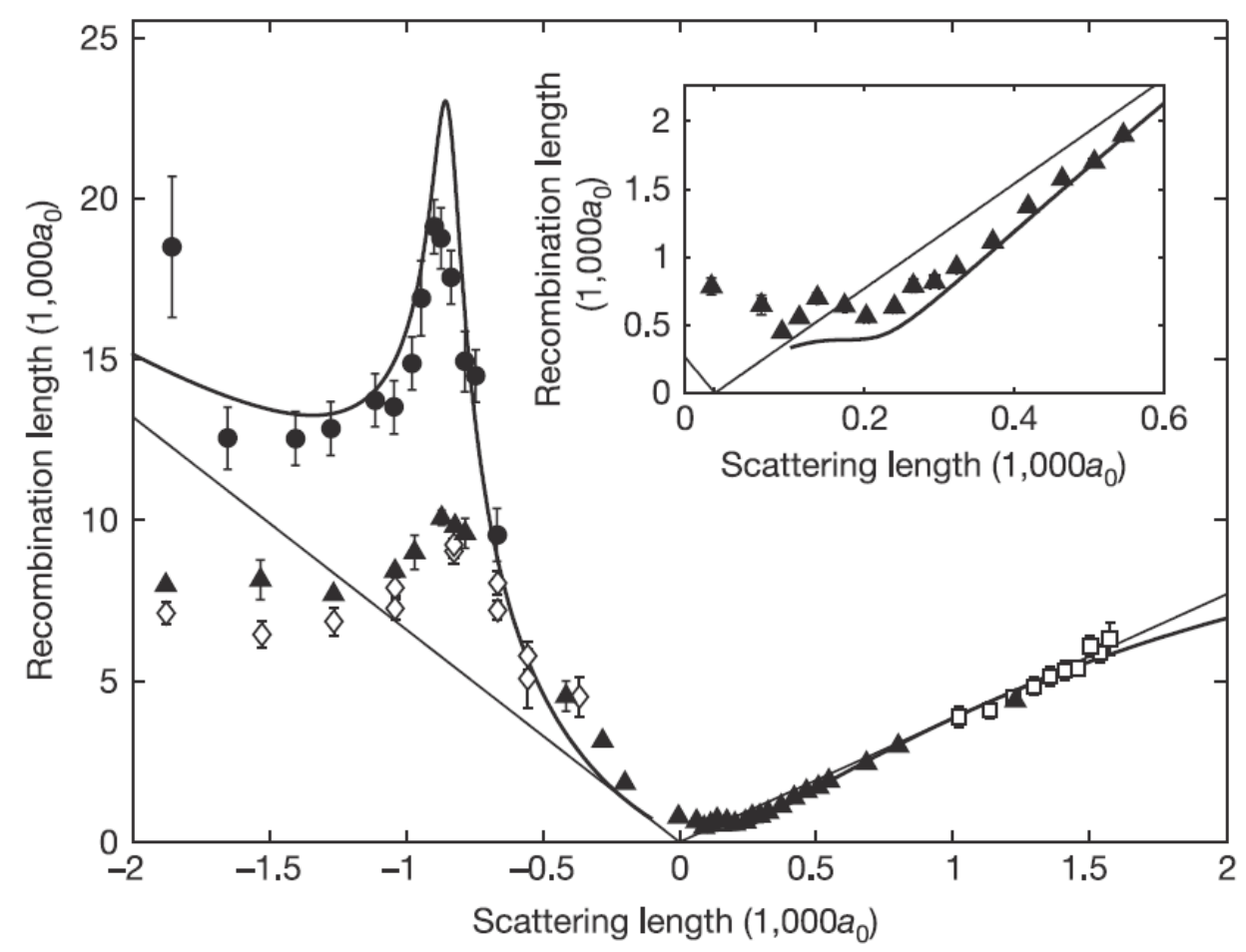

Figure 2 | Observation of the Efimov resonance in measurements of three-body recombination. The recombination length $\rho_{3} \propto L_{3}^{1 / 4}$ is plotted 


\section{Efimov hyperons and delta like}

- vector diquark dissolves more easily than the scalar

- condensed scalars \& zero binding vector diquarks

- $u, d$ and $s$ quarks

- state with zero color, flavor symmetric and spin $3 / 2$

$$
C_{\kappa}=1 \quad \text { (analogous to a 3-boson system) }
$$

DELTA and HYPERONIC LIKE weakly bound/resonant states!!! 


\section{Conclusion}

- Diquarks can be introduced in QCD as an effective degree of freedom (toy model);

- Strong correlation between the quarks - forming diquarks - approaches the fixed point to the real world;

- Near zero-binding diquarks it is possible weakly bound our resonant hyperons and delta-like states;

Next : - Structure of exotic mesons dq - anti dq;

- Nucleon q-dq;

- Thomas-Efimov physics in the formation of strange and non-strange matter...

- extension to other scalar correlations $[q q q q]^{3 c}$

R. L. Jaffe, Phys. Rev. D72 (2005) 074508 